\section{Cahiers de Narratologie}

Analyse et théorie narratives

8 | 1997

Création de l'espace et narration littéraire

\title{
Les nouveaux géomètres. Espaces virtuels et narration littéraire dans La escala de los mapas, de Belén Gopegui
}

\section{Christian Boix}

\section{(2) OpenEdition}

\section{Journals}

Édition électronique

URL : http://journals.openedition.org/narratologie/10723

DOI : $10.4000 /$ narratologie. 10723

ISSN : 1765-307X

Éditeur

LIRCES

\section{Édition imprimée}

Date de publication : 1 décembre 1997

Pagination : 65-79

ISBN : 291089746X

ISSN : 0993-8516

Référence électronique

Christian Boix, "Les nouveaux géomètres. Espaces virtuels et narration littéraire dans La escala de los mapas, de Belén Gopegui », Cahiers de Narratologie [En ligne], 8 | 1997, mis en ligne le 01 décembre 2020, consulté le 25 février 2021. URL : http://journals.openedition.org/narratologie/10723 ; DOI : https://doi.org/10.4000/narratologie.10723

Ce document a été généré automatiquement le 25 février 2021.

Article L.111-1 du Code de la propriété intellectuelle. 


\title{
Les nouveaux géomètres. Espaces virtuels et narration littéraire dans La escala de los mapas, de Belén Gopegui
}

\author{
Christian Boix
}

1 S'il est bien une notion qui représente une primitive sémantique absolue, c'est à n'en pas douter l'ESPACE. Des philosophes aux mathématiciens, en passant par les physiciens et les linguistes, tous s'accordent à placer au cœur de leur réflexion ce soubassement premier. Ainsi par exemple, la sémantique d'inspiration guillaumienne postule une organisation chronologique du sens où l'ESPACE, le TEMPS et la NOTION sont les trois registres ordonnés du sens. De même, la physique moderne nous a appris que le temps ne pouvait se concevoir hors de sa référence à l'espace et que la séparation des deux notions espace/temps n'était possible qu'une fois choisi un référentiel spatial.

2 De là l'importance capitale de cette composante dans le discours - fût-il fictionnel. De notre représentation de l'espace, de la mesure que nous faisons de ce dernier, de "L'échelle des cartes", dirait Belén Gopegui, dépend notre vision du réel, notre interprétation du monde, des autres et de nous-mêmes. Tout changement significatif dans notre culture, dans l'histoire de nos idées, dans nos valeurs éthiques et esthétiques ne saurait se faire sans qu'intervienne une revisitation de cette pierre angulaire dont dépend l'équilibre de l'édifice du sens dans son entier.

3 L'œuvre que nous avons choisie pour éclairer ce postulat nous paraît emblématique d'une évolution rendue indispensable par les cadres nouveaux de ce que l'on nomme du terme commode de "post-modernité $»^{1}$. Face aux apories inquiétantes, ressenties ou pressenties en cette fin de siècle, la réinvention d'une autre logique ne saurait faire l'économie d'une reconstruction du sens. L'émergence de nouveaux arpenteursgéomètres déclarés, significativement jeunes - Belen Gopegui est née en 1963 -, est le signe patent d'une évolution qui intègre les données de la tradition humaniste pour les 
croiser avec les apports de la science moderne : entre le suivisme aveugle et le rejet radical de notre actuel cadre de vie, une voie moyenne ${ }^{2}$, un ESPACE VITAL expérimental est en train de se construire :

Yo tenía un proyecto del que la psicóloga formaba parte y por eso fui a verla. Ella debía proporcionarme la base científica, piedra de toque o roca silícea de color negro contra la que yo frotaría el oro de mi imaginación ${ }^{3}$.

CEuvre au noir des temps modernes, ce projet relève de l'ESPACE VIRTUEL de la création narrative, mais sans doute engage-t-il une révolution plus radicale où la fiction et l'imaginaire ne sont pas d'une nature si éloignée de l'actuelle conception scientifique de l'espace. Ce dernier - fondement du sens, repère initial de nos systèmes astronomique, physique, mathématique - est-il autre chose qu'un effet de notre interprétation? Selon la formule du physicien français Bernard d'Espagnat, «l'espace ne serait qu'un mode de notre sensibilité »...

\section{L'espace «filet du sens »}

Les peuples antiques plantaient des pieux pour marquer leur territoire, aux limites de leurs champs. A travers l'informe de l'étendue, ils disposaient ainsi un réseau d'intelligibilité, une sorte de filet d'intellection jeté sur le monde pour pêcher du sens. Simultanément, il s'agissait d'accomplir un acte magique qui les assurait d'être là : dessiner l'espace c'est se donner le référentiel dont l'être se soutient. C'est ainsi que l'ÊTRE plonge ses racines dans un espace physique (lieu des phénomènes de son activité) que le sujet convertit en espace sensible (celui de ses représentations), avant d'en construire une image théorique qu'il croit dégagée de la contingence, l'espace abstrait des géomètres. Toutefois, par ce filet de signes, marques, lignes, mesures, l'esprit humain ne fait que se renvoyer l'image de sa propre raison. Par la géométrie, l'esprit déchiffre en raison le monde comme une écriture : il crée une algèbre des rapports entre les êtres et entre les choses, entre les êtres et les choses. La géographie, spécialité professionnelle du personnage principal du roman, participe de cette étymologie qui rappelle le transfert symbolique du monde terrestre [Gê, Gea] à l'écriture et au monde des signes [Graphos]. Ainsi avons-nous pris l'habitude de considérer que l'espace est une surface bidimensionnelle englobant habitée par des points et de définir ces points par les rapports abstraits qu'ils établissent sur le vide. Mais qu'arrive-t-il si l'on inverse le rapport algébrique entre plein et vide, ou si l'on suit les scientifiques dans leurs postulats sur un espace vectoriel à trois dimensions, caractérisé par sa courbure ? Cette surprenante question, c'est celle qui est posée - métaphoriquement - dès la première page du roman de Belén Gopegui. Lorsque le géographe Sergio Prim rencontre la psychologue (Maravillas Gea!), son discours accrédite l'idée que le trouble majeur qui affecte sa raison est dû à une hérésie spatiale :

Frente a mi mesa de trabajo hay una ventana que da a un patio interior - dijo en tono reservado -. Es una ventana muy antigua: marco de madera, falleba negra, vidrio esmerilado y amarillo. La falleba se abre girando una manivela que termina en un remate circular, digamos un punto grueso. Si ahora le pido ayuda es porque tal vez decida irme a vivir ahí.

- ¿ Así que piensa irse a vivir a su oficina ? [...]

Sonrió melancólico.

- No, no. Lo importante no es la oficina, sino la manivela. Aunque de todos modos, la manivela es sólo una posibilidad. Hay muchos puntos, huecos, si no tiene inconveniente los llamaremos huecos ${ }^{4}$. 

jeter sur le monde un filet de signes nouveau en questionnant le rapport du vide au plein, du point à l'espace, du plan au creux. De la sorte, remettant en cause ses (et nos) représentations ordinaires de l'espace il propose un nouveau regard porté sur l'ÊTRE. La relation soulignée entre mirar et morar fait ressortir ce rapport du spatial au voir notionnel (comme on parle d'ailleurs de « point de vue ») :

Busqué un hueco. Lo encontré en la tela del abrigo de mi compañero de asiento. Y durante el resto del viaje, moré allí. Mirar, morar. Como ve, una simple vocal puede trastocar la vida de un hombre.

Fuyant le trop-plein du réel moderne, la « superficialité » des cartes qui ont perdu leur sens à force de tenir lieu de réel, cette étonnante fiction narrative propose une reconstruction du palier premier de la conception de l'espace, un voyage aux origines du sens devant permettre une autre appréhension de notre « réel » :

Hubo un tiempo, exagerado y absorto, en que nuestro planeta existió sin mapas. Si un hombre quería representar una región de África en un plano, tenía que ir allí. [...] Mi situación es la misma. Para escribir un tratado del hueco (todos los puntos pertenecen a un único hueco) es imprescindible ir a él. Aún no hay mapas y los escasos testimonios de gentes que dicen haberlo frecuentado son harto imprecisos ${ }^{5}$.

8 En effet, l'agoraphobie de Prim, l'agitation madrilène qui le conduit à se replier sur luimême, sur son appartement, sur ces interstices de calme, ne sont que des représentations - encore spatiales - des formes déstabilisatrices de l'Hybris moderne qui a fait oublier que nos cartes ne constituent jamais que des marques, des traces que l'on a posées sur la manifestation de l'apparence en vue de maîtriser le terrible vertige de l'incompréhensible. Notre époque a fini par se persuader que les pieux habituels que l'homme plante sur le monde sont la nature même, la réalité unique du monde : alors les signes changent de face, au lieu d'être les clefs qui ouvrent la voie, ils deviennent une trame opaque qui barre l'accès au monde. D'où l'ambitieux projet de ce YO narrateur qui est persuadé qu'il faut ré-envisager le soubassement spatial de notre mode cognitif pour imaginer une autre lecture du monde. Il ne s'agit pas de refaire le monde, mais plus modestement de reconsidérer les rapports qui unissent ses composantes : changer d'échelle, c'est changer le monde, puisque nous ne pouvons le voir que par ces médiations spatiales que nous construisons. L'apparente fuite hors de l'espace quotidien est seulement une étape indispensable pour retrouver une science de nos mesures, pour trouver la manière d'arpenter l'étendue (individuelle, sentimentale, sociale, historique) au rythme de notre corps, de notre souffle.

\section{Espace public/espace privé : le faux problème}

La critique contemporaine n'a pas manqué d'insister sur le prétendu désengagement postmoderne, sur le repli d'auteurs incapables de dépasser la sphère du privé, sur le prétendu renoncement implicite au débat éthique. Mais ce changement de cap figuratif peut-il se ramener schématiquement à ce que l'on range avec empressement dans le tiroir de l'«Escapismo"? Là encore, tout se ramène peut-être à une question d'échelle...

Dans La escala de los mapas, la répartition des lieux se fait selon une coupure traditionnelle qui semble à première vue donner prise à des jugements comme celui 
que nous venons de rappeler. L'action des personnages s'inscrit d'un côté sur la carte de l'espace, public et urbain, madrilène :

En aquel momento el 9 abrió sus puertas y apareció Brezo. Escoltada por una piña de viajeros, se bajó del autobús ${ }^{6}$.

$11 \mathrm{Au}$ sein de cet espace englobant, toujours vécu comme une agression par le protagoniste, une première réduction est systématiquement pratiquée. Sergio Prim se défend du monde par un systématique repli sur sa «niche » individuelle. Il se met à l'abri d'un espace extérieur (le monde, les autres) qui est devenu envahissant au point de ne plus laisser la moindre place au soi :

Vivo como ese pequeño país autárquico que ponían de ejemplo en los colegios, soy Albania. [...] En mi república se practica la autarquía de repliegue : producir para autoabas-tecerse y permanecer inmodificado, al abrigo de las influencias extranjeras. Porque habitar con los otros es la guerra y me destruye, he preferido rodearme de una difusa constelación afectiva ${ }^{7}$.

12 Mais ce processus d'apparente césure, s'il en restait là, se contenterait de reproduire un cadre énonciatif préétabli, une scénographie discursive ${ }^{8}$ érigée en genre à une époque donnée. L'originalité de cette œuvre - comme toute production marquante - c'est de se poser en excès de ce sur quoi elle s'appuie. Le repli maniaque sur un espace individuel se résoudra ici en une curieuse quête de points et de vides, quête d'un espace infinitésimal au sein duquel le sujet cherche à ouvrir des brèches d'espaces insoupçonnés :

Según he comprobado, los objetos esconden una concavidad invisible?.

Il ne s'agit plus simplement de se replier sur soi : décider d'aller vivre dans l'extrémité d'une manivelle, comme Sergio Prim l'envisage dès la première page du roman, c'est une aventure, un "dépaysement " qui fait courir dans la masse compacte des signes fossilisés une fracture par où, peut-être, l'étrangeté d'une issue va pouvoir s'introduire. Parmi tant d'objets, de signes, d'images, d'idées, d'activités qui encombrent l'espace extérieur, il devient vital de trouver quelques «trous » où glisser l'espace intérieur de l'imagination humaine, lequel n'a plus - littéralement - droit de cité. L'espace extérieur public, le contenant surdimensionné, a totalement obéré la place devant revenir à un espace intérieur privé, contenu sous-dimensionné. D'où la réflexion sur la nécessité de revoir nos mesures, l'échelle de nos cartes : transformer un monde de repères, c'est échapper à une logique pour en construire une autre. Renverser l'échelle, c'est opérer une véritable "révolution culturelle», mentale cette fois. Il n'est pas surprenant de trouver dans ce livre, en exergue d'un chapitre central, un fragment du Livre du Tao :

Treinta radios convergen en el centro de una rueda,

pero es su vacío

lo que hace útil al carro.

Se moldea la arcilla para hacer la vasija,

pero de su vacío

depende el uso de la vasija ${ }^{10}$.

14 Ces vides, désignés par le plein, sont déclinés par le roman selon une grande variété d'images :

- ¿Conoces - pregunté - esta frase de Claude Debussy ? : « la música no está en las notas, sino entre las notas $»^{11}$. [...]

Invéntame una barra espaciadora como la de las máquinas de escribir pero más grande, que sirva para la vida ${ }^{12}$. 
La materia no es compacta, como saben. Entre el núcleo del átomo y su electrón queda sitio, y entre una molécula y otra. La materia es discontinua, como la energía y, según ciertas hipótesis, también lo son el espacio y el tiempo ${ }^{13}$.

Cet espace « inventé » par agrandissement d'échelle, est le dernier lieu, le dernier défi que l'esprit peut se lancer à lui-même. Après des siècles de réflexion à deux dimensions, fondée sur les surfaces et les lignes, voici venu le temps - confirmé par les sciences dures - des trous, des courbes et des creux, des vides habités ${ }^{14}$. Aux dires du narrateur, l'échec antérieur, les erreurs passées ne laissent plus que cette ultime possibilité :

[...] he venido a parar a este retículo cero de la Tierra, lugar exento de equivocaciones cuyo retrato me incumbe como una deuda de honor, como una última responsabilidad ${ }^{15}$.

Toutefois, comme si la narration voulait prévenir toute interprétation réductrice de la métaphore de cette recherche spatiale, elle se réfère elle-même à la terminologie critique consacrée pour désigner toute œuvre ne respectant pas l'échelle dite « réaliste » des représentations romanesques :

Con tono lóbrego, me remonté a los últimos años de colegio, cuando procuraba esconderme de todos los demás. Me iba solo durante los recreos hasta que oía una voz: « ¡ Eh tú ! ¡Sí, es a ti !», y yo me quedaba quieto : « ¡ESCAPISTA!», y yo me daba la vuelta ${ }^{16}$.

7 De ce récit d'un passé révolu par rapport au présent diégétique, on peut aisément déduire un humour distancé qui affirme que nous sommes maintenant parvenus à un âge adulte qui va au-delà d'une simple fuite irresponsable. A plusieurs reprises, le narrateur s'amuse de cette vision critique simpliste portée sur la littérature « dégagée de la présence du référent historique ${ }^{17} »$ :

Brezo me oponía tesis pragmáticas : «Si por lo menos te fueras, si un día lo hicieras - solía decirme - ; LO PEOR DE LOS ESCAPISTAS ES QUE NUNCA SE ESCAPAN DE VERDAD $^{18}$.

8 Du point à la surface, du particulier au général, de l'imaginaire au réel, il n'y a qu'un changement d'échelle, un changement algébrique dans notre appareil de mesure interprétative du réel :

- Tú sabes que las escalas no son patrimonio de los geógrafos. En realidad, todo el mundo los utiliza para interpretar los datos que tiene. Por ejemplo, el otro día estaba en la cocina y, a través de la ventana del patio, escuché un rebullir oscuro : podía ser la cafetera de una casa, el café subiendo, o un avión. El sonido era idéntico. Simple cuestión de escala ${ }^{19}$.

Sous cette perspective, la médiation des signes de la fiction, l'imagination narrative, dessinent les cartes de l'humain selon le même principe qui permet aux géographes de rendre compte du monde. Il n'y a donc pas de différence de nature entre la mesure du monde et celle de l'homme, et toute représentation forgée pour parler de l'un parle inévitablement de l'autre. Seule une vision séparant contenant et contenu, plein et vide, spatial et notionnel, broie le continu universel dans l'émiettement de signes opaques qui nous éloignent de l'UN, dont l'incarnation est dans l'ESPACE. La proposition de l'espace romanesque de La escala de los mapas consiste à ré-envisager les rapports de l'infiniment grand et de l'infiniment petit, la frontière factice de l'intérieur et de l'extérieur. Trouver une nouvelle unité de mesure, une nouvelle échelle, c'est trouver le dénominateur commun permettant de situer également le SOI, les AUTRES, et le MONDE que nous habitons. Là est le défi. Trouver la voie d'accès conduisant « De l'autre côté du miroir »: 
Todo está comunicado : yo guardo un bolígrafo en el cajón y, como el conejo de Alicia, puede el bolígrafo llegar al jardín de la reina [...] $]^{20}$.

\section{L'esthétique d'une révolution éthique : l'espace virtuel comme demeure de l'imagination créatrice}

La escala de los mapas renvoie à l'espace originel sur lequel nous érigeons nos cartes. Le premier, comme les secondes par lesquelles il advient à l'existence mentale, trouvent leur origine première et absolue dans le pouvoir de l'imagination humaine. D'où la propension à jouer d'abondance de la catégorie des ESPACES VIRTUELS. C'est ainsi que la « rencontre amoureuse » sur laquelle se fonde l'argument a tous les dehors de l'image virtuelle en trois dimensions, de l'animation qui produit du réel à partir du presque rien de l'idée. Sergio Prim rencontrera Brezo en un lieu peu orthodoxe, comme le précise énigmatiquement le narrateur à l'intention d'un destinataire collectif qui ressemble fort aux lecteurs :

Verán, yo encontré a Brezo en una suposición. [...] La imaginé camino de su casa por la calle de Alcalá e imaginé que ella reconociá mi silueta bajo el alero de la parada del autobús ${ }^{21}$.

De surcroît, les personnages eux-mêmes sont susceptibles de déroger aux lois premières du vraisemblable réaliste. En effet, au début de la narration, le narrateur focalisateur est censé être une psychologue, s'exprimant à la première personne, qui reçoit à son cabinet un certain Sergio Prim, géographe. Toutefois, cinq pages à peine après le début du livre, nous faisons cette surprenante découverte :

Me tranquilicé. Sergio Prim tenía la expresión grave y no mentía. Sergio Prim no mentía porque yo soy Sergio Prim ${ }^{22}$.

Notre sentiment ordinaire de la permanence d'un espace-temps de référence subit d'entrée un choc déstabilisateur. Nous avons zappé d'un référentiel à un autre, nous sommes passé d'un schéma virtuel à un autre schéma tout aussi virtuel. Néanmoins, contrairement à une perception appauvrie du sens de l'adjectif «virtuel ", un espace ainsi qualifié n'est pas forcément un leurre qui s'opposerait à l'évidence d'un espace réel et falsifierait ce dernier. L'espace virtuel (espace ordinaire de La escala de los mapas) est un lieu où l'imagination est la source reconnue - et assumée en pleine responsabilité - d'une réalité alternative, d'un monde parallèle tout aussi efficient que celui que dessinent les repères habituels :

Estábamos en octubre, pero a mí me vino a la cabeza el ambiente de las hileras de bombillas y árboles encendidos que hay en las Navidades de Madrid. Inmediatamente pensé : "A lo mejor ha vuelto ", pues cada año, estuviera donde estuviera, Brezo regresaba para pasar la Nochebuena con su padre viudo ${ }^{23}$.

En effet, est VIRTUEL ce «qui a en soi toutes les conditions essentielles à sa réalisation ${ }^{24}$ ", ce qui peut aussi bien accéder à l'existence. Les dictionnaires attestent tous de l'échelle de progression : possible $\rightarrow$ probable $\rightarrow$ virtuel, le dernier terme étant celui de la limite fragile qui jouxte l'espace de ce que nous appelons « réel ». Du réel au virtuel, il n'existe qu'une « membrane poreuse » dans laquelle se trouvent peut-être ces «trous » dont parle sans cesse Sergio Prim... Toujours est-il que la création fictionnelle de Belén Gopegui nous place devant un univers où la loi du vraisemblable vise à abolir la frontière entre les espaces empreints de "réalisme " (rues de Madrid, bars, dates, types humains et fonctions professionnelles) et les espaces virtuels issus de l'imagination. Dans ces espaces parallèles peut se développer une méthode expérimentale, 
très certainement aussi stimulante pour la littérature et pour l'esprit qu'avait pu l'être en son temps la méthode d'observation du même nom qui avait inspiré le Naturalisme d'un Zola. Simplement, les temps ayant changé, les méthodes expérimentales $s^{\prime}$ appuient sur de nouveau outils ${ }^{25}$ :

Un experimento consiste en PROVOCAR cierto fenómeno para estudiarlo, y ustedes me preguntarán cómo se puede provocar un hueco. También Brezo me lo preguntó. Supón, le dije, que... [.... $]^{26}$.

C'est bien sans doute ce changement d'époque et de méthode que veut souligner le narrateur lorsqu'il ajoute incidemment, lors d'une description, trois pages après les lignes que nous venons de citer :

Era aquel tren CONTRARIO A CUALQUIER RELATO DECIMONÓNICO ${ }^{27}$.

Mais précisément, comment transposer cette nouvelle démarche, dont l'espace virtuel est le premier palier et l'outil décisif, au domaine de l'Être ? Transférer le vécu à la page d'écriture, représenter, fait partie de nos habitudes mentales inculquées dès le plus jeune âge, mais comment faire accéder cette liberté imaginant à l'actualisation, comment réaliser la démarche inverse à celle d'une esthétique simplement descriptive?

Nadie me puso ejercicios para aprender a trasladar lo imaginado a lo vivido. Escribir en el día lo que habíamos escrito en el cuaderno; nadie me hizo caso en clase cuando sugerí que hiciéramos redacciones al revés ${ }^{28}$.

Percevoir et décrire l'espace comme un déjà-là, c'est dresser un état des lieux préexistant. Produire des espaces virtuels par le biais de l'imagination, c'est enrichir la future carte de données nouvelles, c'est ouvrir des potentialités : ces espaces sont la demeure potentielle de nouveaux espaces éthiques que l'on expérimente, dont on éprouve la viabilité. La création narrative est déjà une manière de les faire advenir à l'existence. Sans doute l'espace de l'art, de l'écriture, l'impalpable réseau de la communication universelle - celui du partage des espaces de l'imagination - est-il le seul vecteur propice à faire advenir une « rédaction à l'envers » :

Es preciso conectarse, Brezo, mira que sucesión de antenas por los tejados ${ }^{29}$.

Y pensé en nosotros, en todos nosotros, muchachas de terciopelo, hombres que tienen un soplo en el corazón : óiganme, haremos que se establezca un itinerario paralelo ${ }^{30}$.

Tous ceux qui croient en la littérature, cette " delicada pléyade de introvertidos » ${ }^{31}$ qui habitent ces espaces laissés vides par les autres, qui goûtent à l'esthétique du virtuel parce qu'ils souscrivent à l'éthique d'un univers parallèle construit par l'Homme à la mesure de l'Homme, sont susceptibles de cartographier autrement le monde. Et alors tout redevient possible. Sous nos yeux la "réalité » se déforme sous la nouvelle échelle et change de visage :

Veríamos entonces cambiar la geometría de las plazas, multiplicarse la distancia entre las Cibeles y la Puerta del Sol, crecer la densidad de población de África, la extensión de China, la altitud del Retiro ${ }^{32}$.

Si sobre un atlas abierto en la página del mapamundi dejases caer un trozo de corteza de pan, verías, Brezo, temblor, extravío, descarrío de mi alma, que ocupa lo mismo que Dinamarca ${ }^{33}$.

Si l'Être humain peut avoir une force indestructible, un pouvoir sur l'univers, c'est depuis ce lieu fondamental de la création, de l'imagination, de la mesure dont il fixe 
librement l'échelle. Seul l'espace des signes grâce auxquels il balise son réel vertigineux donne à l'Être la sensation d'une permanence, d'un sens.

Ce lieu, qui ouvre des espaces possibles dans la trame compacte et fuyante du monde pour le rendre habitable est bien celui de l'ÉCRITURE, où l'on se raconte l'univers, comme en attestent les dernières lignes du roman de Belén Gopegui :

$\mathrm{Y}$ así yo, desde la primera letra, sigo aquí, no me he movido. Al fin cambié la escala $\mathrm{y}$ vine a quedarme en este espacio iluminado. Doscientas veintinueve páginas rectangulares con inscripciones impresas, y entre cada palabra, y al borde de cada letra, un intervalo, un hueco. Alza la mano y verás cómo el espacio se detiene ${ }^{34}$.

Nous trouvons au terme du parcours une vision de la sagesse dans un monde au repos, caractérisé par la cessation du flux (alza la mano y verás cómo el espacio se detiene), un monde où le sujet se confond avec la totalité (desde la primera letra sigo aquí, no me he movido) de l'espace et des éléments.

31 Cette abstraction métaphorique continue que nous présente La escala de los mapas rejoint très exactement la tradition bouddhique de la " voie moyenne», laquelle permet de postuler une éthique du "sans fond » où l'individu découvre la nécessité d'élargir son horizon pour y introduire des approches transformatrices de l'expérience. Il ne s'agit pas de mettre en lumière l'espace d'un fondement absolu ni de découvrir un soi caché, mais bien plutôt de rejoindre la voie d'une empathie spontanée. Car il n'y a sans doute rien en-deçà de notre EXPÉRIENCE IMAGINANTE DES RÉGIONS DE L'ESPACETEMPS que nous habitons. Elle est notre richesse infinie si nous voulons bien lui prêter toute l'attention qu'elle mérite et cesser de nous aveugler en lorgnant du côté d'une « réalité » fondatrice introuvable.

Mais qui croirait un géographe à moitié fou, imprégné de culture scientifique moderne, versant, comme nombre de physiciens et penseurs contemporains, dans la philosophie orientale ${ }^{35}$, chétif et introverti, double d'un YO narrateur qui ne vit que par, dans et entre les signes d'un roman? Peut-être ceux qui ont partagé sa vie précaire, l'espace d'une lecture... C'est, en tout cas, à ce travail de persuasion, de conversion à un acte de foi, que s'est attelée cette innovante fiction :

Realidad, santo cielo, qué vulgares son tus tropas. Traté de hacerles ver que el presente no es sólo esta sucesión de cuerpos pesados con su carne y sus huesos, cubos y esferas, líquidos y sólidos ${ }^{36}$.

\section{NOTES}

1. Pour une mise en perspective de cette question, cf. C. BOIX : «La fin du XXe siècle. Déni de l'histoire ou changement de paradigme?", in Notre fin de siècle. Culture hispanique, Dijon, Hispanística XX, Centre d'Études et de Recherches Hispaniques du XXe siècle, Université de Bourgogne, 1996 (p. 27-46).

2. Cette expression est à prendre au sens que lui donne Francisco VARELA, à savoir la tentative d'une médiation entre nos conceptions scientifiques de la vie et de l'esprit, d'une part, et notre autocompréhension quotidienne, vécue. Cf. F. VARELA et al. : L'inscription corporelle de l'esprit, Paris, Seuil, 1993. 
3. B. GOPEGUI : La escala de los mapas, Barcelona, Anagrama, 1993, p. 15.

4. Ibid., p. 9-10.

5. Ibid., p. 12.

6. Ibid., p. 16.

7. Ibid., p. 29.

8. Pour une définition de ce concept, $\mathrm{cf}$. D. MAINGUENEAU : Le contexte de l'œuvre littéraire, Paris, Dunod, 1993, p. 121-135.

9. B. GOPEGUI, op. cit., p. 11.

10. Ibid., p. 100.

11. Ibid., p. 55.

12. Ibid., Id.

13. Ibid., p. 102.

14. Les références aux mathématiques, à la physique quantique, à l'astronomie, sont légion dans le roman. Belén Gopegui introduit dans sa réflexion humaniste les représentations des sciences contemporaines. Cette posture consacre un tournant qui mérite d'être salué: le roman contemporain va peut-être sortir des cadres immuables du XIXe...

15. B. GOPEGUI : op. cit., p. 15-16.

16. Ibid., p. 32.

17. Nous empruntons cette expression au livre de B. BESSIERE: Vingt ans de création espagnole. 1975-1995, Paris, Nathan Université, col. 128, 1995, p. 50.

18. B. GOPEGUI : op. cit., p. 32. [Souligné par nous]

19. Ibid., p. 127.

20. Ibid., p. 131.

21. Ibid., p. 16. [Souligné par nous]

22. Ibid., p. 14.

23. Ibid., p. 16.

24. Le Petit Robert : entrée « Virtuel ».

25. Le formidable outil scientifique de la simulation sur ordinateur n'est rien d'autre qu'une expérimentation sur des espaces virtuels, à partir desquels on analyse du réel. La science a bien su trouver des «trous » dans la membrane de séparation virtuel/réel...

26. B. GoPEGUI, op. cit., p. 109. [Souligné par nous]

27. Ibid., p. 112. [Souligné par nous]

28. Ibid., p. 169.

29. Ibid., p. 173.

30. Ibid., p. 219.

31. Ibid., id.

32. Ibid., p. 85. Cette anamorphose n'est pas le seul fruit d'une imagination littéraire délirante. La pointe de la recherche actuelle en géographie propose des modèles cartographiques qui tentent de s'affranchir de l'espace bidimensionnel des cartes d'antan pour traduire les données sous la forme de représentations nouvelles. La très sérieuse directrice du laboratoire Image et ville de l'université de Strasbourg, Colette Cauvin, n'hésite pas à transgresser les normes les plus usitées : "Pour moi, la géographie est une manière de traiter les données, de révéler par une carte quelque chose qu'on ne voyait pas, une dimension invisible. » Ainsi, l'espace n'est pas forcément mesuré en kilomètres. Il peut l'être en temps ou en coût de transport, voire en intentions de vote. Ses représentations prennent alors un relief singulier : le planisphère des populations montre ainsi une Inde gigantesque par rapport à une minuscule Australie. Par contre, l'île-continent va faire figure de géant si la variable retenue est le produit intérieur brut par habitant. Les géographes embrassent maintenant des disciplines de plus en plus nombreuses. Dans le souci de faire parler toujours mieux les cartes de synthèse de leurs travaux ils envisagent de passer à une 
animation de leurs graphiques: et si les images se déformaient sur un écran, racontant par exemple l'impact d'une règlementation sur l'implantation des magasins à grande surface, ou celui d'une taxe sur la consommation de carburant, ... ou celui du surgissement d'une aventure amoureuse dans la morne plaine de l'existence de Sergio Prim? [Source: Publications URA 902 - CNRS - Image et Ville - Université de Strasbourg]

33. B. GOPEGUI, op. cit., p. 227.

34. Ibid., p. 229.

35. Le patronyme de Brezo, à savoir VARELA, est susceptible de renvoyer aux écrits de Francisco Varela, directeur de recherche au CNRS et membre du CREA de l'École Polytechnique de Paris. La convergence de ses positions philosophiques avec les développements présents dans La escala de los mapas est, en tout cas, troublante...

36. B. GOPEGUI, op. cit., p. 228.

\section{AUTEUR}

\section{CHRISTIAN BOIX}

Université de Toulouse II 